\title{
Analysis of tape tether survival in LEO against orbital debris
}

\author{
Shaker Bayajid Khan*, Juan R. Sanmartin
}

\begin{abstract}
The low earth orbit (LEO) environment contains a large number of artificial debris, of which a significant portion is due to dead satellites and fragments of satellites resulted from explosions and in-orbit collisions. Deorbiting defunct satellites at the end of their life can be achieved by a successful operation of an Electrodynamic Tether (EDT) system. The effectiveness of an EDT greatly depends on the survivability of the tether, which can become debris itself if cut by debris particles; a tether can be completely cut by debris having some minimal diameter. The objective of this paper is to develop an accurate model using power laws for debris-size ranges, in both ORDEM2000 and MASTER2009 debris flux models, to calculate tape tether survivability. The analytical model, which depends on tape dimensions (width, thickness) and orbital parameters (inclinations, altitudes) is then verified with fully numerical results to compare for different orbit inclinations, altitudes and tape width for both ORDEM2000 and MASTER2009 flux data.
\end{abstract}

(C) 2014 COSPAR. Published by Elsevier Ltd. All rights reserved.

\section{Introduction}

Deorbiting defunct satellites at the end of their life to reduce the chances of Kessler cascading (Kessler and Cour-Palais, 1978) can be achieved by a successful operation of an Electrodynamic Tether (EDT) system. An EDT works on the basis of the interaction between the electric current flowing along the tether and the geomagnetic field, which produces a Lorentz drag force, the current being itself induced by the tether motion relative to the Earth's magnetized ionosphere; this reflects on the thermodynamic character of that interaction, always leading like air-drag, to a decrease in the relative motion of tether and ambient plasma. For more than a decade till now different studies have been carried out on various tether con- figurations and deorbiting performance (Forward et al., 1998; Vannaroni et al., 1999; Van der Heide and Kruijff, 2001; Ahedo and Sanmartin, 2002; Gilchrist et al., 2002; Nishimine, 2002; Pearson et al., 2003), providing a propellantless, cost-effective solution to deorbiting dead satellites as well as future satellites after their desired work-life. The bare tether concept, which has the tether bare of insulation, collecting electrons itself, has eliminated the need for a plasma contactor at the anodic end (Sanmartin et al., 1992; Sanmartin et al., 1993).

The low earth orbit (LEO) region, being itself congested by numerous artificial debris caused by space exploration for the last 60 years, poses a risk to safe operation of an EDT, which, if cut due to collisions with particles, can itself be a kind of debris for other operating satellites. The collision risks are more likely to occur with smaller objects than with comparatively larger ones as the flux of larger objects at LEO altitudes is significantly low. Previous analysis showed that a tape performs much better than a round 
tether at survival against debris impact (Khan and Sanmartin, 2013). For given time in orbit, a tape experiences a number of fatal impacts about one and a half orders of magnitude lower than a round tether of equal mass and length. Because deorbiting from a given altitude is faster for the tape due to its larger perimeter, its probability of survival in a practical sense is even higher. Objective of the present study is deriving an approximate analytical model to calculate tape survival probability at given conditions, based on man-made debris flux represented by both NASA and ESA models, calculated for the altitude range $250-1500 \mathrm{~km}$, different inclinations and different values of width and thickness; this simple model should be useful for preliminary design on tape dimensions for satellite deorbit missions. A second objective is to check the accuracy of the analytical model by comparing its results with fully numerical computations from the NASA (ORDEM2000(Liou et al., 2002)) and ESA (MASTER2009 (Flegel et al., 2009)) flux data.

Sections 2 and 3 present models for fatal impact rate and cumulative debris flux data (as presented by ORDEM2000 and MASTER2009) respectively. Results are compared with fully numerical computations in Section 4, and then Section 5 summarizes basic conclusions.

\section{Fatal impact risk modeling}

For a tether while deorbiting, collision risk is roughly defined as the product of tether-debris effective collision area, deorbit time and the concerned flux of particles. One needs taking into account the macroscopic size of debris while calculating collision cross-section area, as debris, representing a potential threat on severing a tether, do have considerable size compared to tether width. The Poisson distribution gives the tape survival probability which, if high, is just $e^{-N_{0}} \simeq 1-N_{c}$, where $N_{c} \ll 1$ is the number of fatal impacts. The small-debris fatal impact rate per unit time and tape length $L$ can be written as (Khan and Sanmartin, 2013)

$$
\begin{aligned}
& \frac{N_{c}}{L \Delta t} \cong \dot{n}_{c}=\int_{0}^{\frac{\pi}{2}} \frac{\mathrm{d} \theta}{\pi / 2} \mathrm{I}(\theta) \\
& I(\theta)=\int_{\delta_{m}}^{\delta_{\infty}} \frac{-\mathrm{d} F}{\mathrm{~d} \delta} \mathrm{d} \delta \widetilde{D}_{e f f}
\end{aligned}
$$

as extension of the usual estimate for round tethers (Pardini et al., 2007; Pardini et al., 2009), with $F(\delta)$ cumulative debris-flux down to a generic debris size $\delta$, tether resident time in orbit $\Delta t$, and $\theta$ impact angle relative to normal to the wide side of the tape. Also $\delta_{m}(\theta)$ is the lower bound in the $\delta$-integral in Eq. (1b), the upper bound $\delta_{\infty}$ being any large debris size, say $1 \mathrm{~m}$. $\widetilde{D}_{\text {eff }}(\delta, \theta)$ is an effective (equivalent) "diameter" for collisions.

Energy considerations and empirical results show that hypervelocity impact of debris particles on a surface excavate hemispherical pits/craters or puncture holes depending on the thickness and material of the exposed surfaces.
The size of excavated hemispherical pits/craters can be several times the diameter of the impactor particle (Hayashida and Robinson, 1992; Ryan and Christiansen, 2010; Simon et al., 1993; Francesconi et al., 2012), depending on the impact velocity as well as materials properties but the value 3 times is considered representative. However, if the material behind the surface is thin (like in our case, a tape) relative to the diameter of the debris particle, the impact actually produces a punctured hole about the size of the impactor; this is because the particle is not allowed sufficient time to get very hot, which disrupts the compression wave by a propagating shock (Hastings and Garrett, 1996).

In the case of a round tether of diameter $D$, the excavated volume was to be equated to a cut-off chunk of tether,

$\frac{1}{2} \cdot \frac{4}{3} \pi\left(\frac{3 \delta_{m}}{2}\right)^{3}=\delta_{m} \pi D^{2} / 4 \Rightarrow \delta_{m}=\frac{D}{3}$

Further, one introduces an effective diameter (front area per unit length), $D_{\text {eff }}=\delta+D-\delta_{c}$, which takes into account the need for debris and tether volumes to overlap in fatal collisions. The overlap is represented by a fraction of diameter, typically taken as $\delta_{c}=\delta_{m}$, yielding $D_{\text {eff }}=\delta+2 D / 3$ (Kessler, 2000).

For a tape with width $w$ and thickness $h$, the front area per unit length ("diameter") is

$\widetilde{D}(\theta)=w(\cos \theta+\epsilon \sin \theta) \equiv w_{\theta}^{\prime}, \quad \epsilon \equiv h / w$

leading to an effective (equivalent) "diameter",

$\widetilde{D}_{e f f}(\delta, \theta)=\delta+w_{\theta}^{\prime}-\delta_{c}, \quad \delta_{c}=w_{\theta}^{\prime} / 3$

As regards $\delta_{m}$ there is a basic difference with the round tether case. As Eq. (2) we would now have,

$\frac{1}{2} \cdot \frac{4}{3} \pi\left(\frac{3 \delta_{m}}{2}\right)^{3}=\delta_{m} w h \Rightarrow \delta_{m} \propto \sqrt{w h}$

which is much less than $\delta_{c}=w_{\theta}^{\prime} / 3$ except for $\theta$ very close to $\pi / 2$ (glancing impact, see Fig, 1). This basic differencereflects on the fact that a debris of size $\sim \sqrt{w h}$ just punctures a hole and leaves almost no energy in the tape material. For a tape, away from glancing incidence, $\delta_{m}$ is taken as equal to $\delta_{c}=w_{\theta}^{\prime} / 3$ as required by the need to provide volume overlap,

$\delta_{m}(\theta)=\frac{1}{3} w_{\theta}^{\prime} \quad$ for $\theta \leqslant \theta_{g l}$

where $\theta_{g l}$ is the angle marking the start of glancing incidence, where both conditions Eq. (5) and Eq. (6) hold. Using Eq. (6) in Eq. (5),

$\frac{1}{2} \cdot \frac{4}{3} \pi\left(\frac{3 w_{\theta}^{\prime} / 3}{2}\right)^{3}=\frac{w_{\theta}^{\prime}}{3} w h \Rightarrow \cos \theta_{g l} \approx \sqrt{4 \epsilon / \pi}$,

where we used $\cos \theta \gg \epsilon$ from Eq. (7) itself, finally yields,

$\delta_{m}(\theta)=\delta_{g l}=\frac{1}{3} \sqrt{\frac{4}{\pi}} \sqrt{w h} \quad$ for $\theta>\theta_{g l}$ 


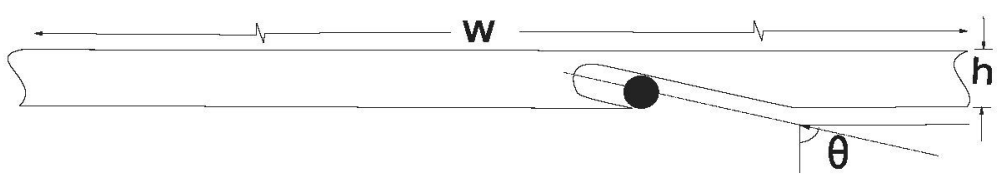

Fig. 1. Glancing incidence, impact angles corresponding to $\theta>\theta_{g l}$.

For debris flux $F$ we shall use separately ESA's Meteoroid and Space Debris Terrestrial Environment Reference (MASTER2009) model and NASA's Orbital Debris Engineering Model (ORDEM2000), which is comparatively conservative, both allowing for simple analyses. Using $\widetilde{D}_{\text {eff }} \approx \delta_{\infty}$ at $\delta=\delta_{\infty}$, the $\delta$-integral of Eq. (1b) can be rewritten as,

$I(\theta)=w_{\theta}^{\prime} F\left(\delta_{m}\right)-\delta_{\infty} F\left(\delta_{\infty}\right)+\int_{\delta_{m}}^{\delta_{\infty}} \mathrm{d} \delta F(\delta)$

where the second term on the right hand side is very small and hence neglected afterwards.

\section{Debris flux modeling}

Predicted flux values by MASTER2009 are almost one order of magnitude lower than ORDEM2000 in the diameter range of interest of around $1 \mathrm{~mm}$, for typical values $\sqrt{w h} \sim$ $1 \mathrm{~mm}$. For $\delta>10^{-4} \mathrm{~m}$, the ORDEM2000 flux, regardless of orbit inclination, can be accurately approximated as three power laws meeting at points $\delta^{*}, F^{*}$ and $\delta_{*}, F_{*}$ (Fig. 2), which is also reasonably valid for MASTER2009.

The Power law approximations of flux for three different diameter ranges can be written as,

$F(\delta) \simeq\left\{\begin{array}{l}F_{0}(\delta) \equiv F^{*}\left(\delta^{*} / \delta\right)^{n_{0}}, \quad \delta<\delta^{*} \simeq 1 m m \\ F_{1}(\delta) \equiv F^{*}\left(\delta^{*} / \delta\right)^{n_{1}}=F_{*}\left(\delta_{*} / \delta\right)^{n_{1}}, \quad \delta^{*}<\delta<\delta_{*} \\ F_{2}(\delta) \equiv F_{*}\left(\delta_{*} / \delta\right)^{n_{2}}, \quad \delta_{*}<\delta\end{array}\right.$

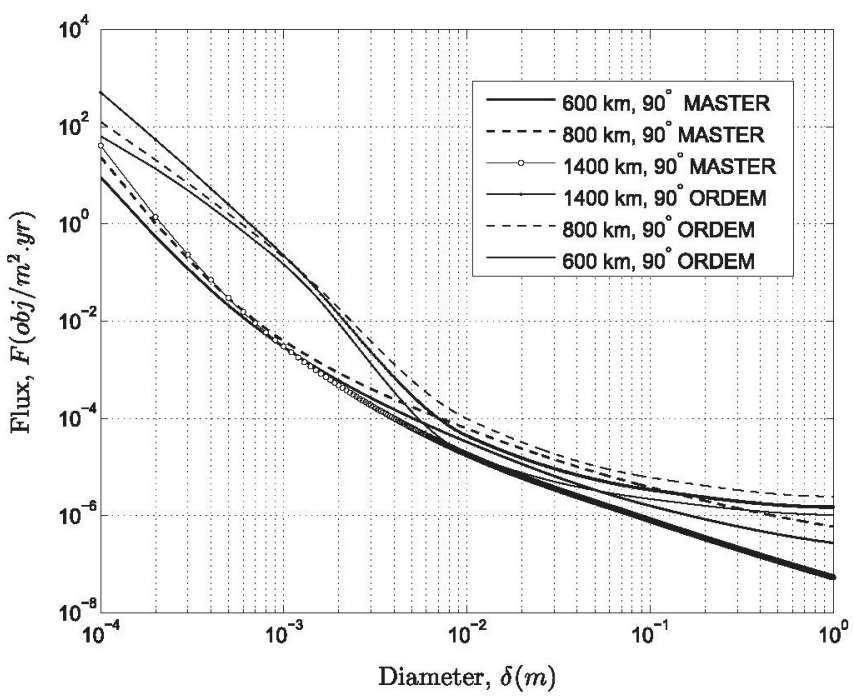

Fig. 2. Flux vs. debris diameter (ORDEM2000 and MASTER2009), for year 2013 .
Typically, $\delta_{*}$ lies around a value $2 \mathrm{~cm}$, which also lies around the maximum width for the Orbital Motion Limited (OML) current law for a cylindrical probe to hold at typical Debye lengths (Sanmartin and Estes, 1999), and $\delta^{*}$ values range over $1 \mathrm{~mm}$, except at altitudes 825 $950 \mathrm{~km}$ for ORDEM2000 data.

In Eq. (10) $n_{0}, n_{1}$, and $n_{2}$ are the three approximate slopes for flux vs. debris diameter in log $\log$ scale, for the aforementioned diameter ranges. Actually, MASTER2009 barely shows a break in power law at $\delta_{*}, F_{*}$, with $n_{2} \simeq n_{1}$ (Fig. 2). Further, for both MASTER2009 and ORDEM 2000, the flux at $\delta>\delta_{*}$ is already very low and contributes negligibly to $I(\theta)$ in Eq. (9), the $n_{2}$ power law dropping from the analysis altogether. Fig. 3 presents $n_{0}, n_{1}$, and $\delta^{*}(\mathrm{~cm})$ versus orbital altitude $H$ showing very little dependence on orbital inclination. Representative $F^{*}$ (and $F_{*}$ ) values cover a large range, however.

Assuming $\delta_{m}(\theta)<\delta^{*}$ we can write Eq. (9) as,

$\frac{I(\theta)}{\delta^{*} F^{*}} \simeq \frac{w_{\theta}^{\prime}}{\delta_{m}} \frac{\delta_{m} F_{0}\left(\delta_{m}\right)}{\delta^{*} F^{*}}+\int_{\delta_{m}}^{\delta^{*}} \frac{\mathrm{d} \delta F_{0}(\delta)}{\delta^{*} F^{*}}+\int_{\delta^{*}}^{\delta_{*}} \frac{\mathrm{d} \delta F_{1}(\delta)}{\delta^{*} F^{*}}$

where we already ignored the $F_{2}$ integral from $\delta_{*}$ to $\delta_{\infty}$. Carrying out the two integrals in Eq. (11), we find

$$
\begin{aligned}
\frac{I(\theta)}{\delta^{*} F^{*}}= & \left(\frac{w_{\theta}^{\prime}}{\delta_{m}}+\frac{1}{n_{0}-1}\right)\left(\frac{\delta^{*}}{\delta_{m}}\right)^{n_{0}-1}-\frac{n_{1}-n_{0}}{\left(n_{1}-1\right)\left(n_{0}-1\right)} \\
& -\frac{1}{n_{1}-1}\left(\frac{\delta^{*}}{\delta_{*}}\right)^{n_{1}-1}
\end{aligned}
$$

where the last term can also be ignored.

As regards the $\theta$-integral, we write Eq. (1a) as

$\dot{n}_{c}=\int_{0}^{\theta_{g l}} \frac{\mathrm{d} \theta}{\pi / 2} I\left(\theta \leqslant \theta_{g l}\right)+\int_{\theta_{g l}}^{\frac{\pi}{2}} \frac{\mathrm{d} \theta}{\pi / 2} I\left(\theta>\theta_{g l}\right)$

and using Eqs. (6) and (8) for $\delta_{m}$ in Eq. (12), we finally obtain,

$$
\begin{aligned}
\left(\frac{w}{3 \delta^{*}}\right)^{n_{0}-1} \frac{\pi}{2} \frac{\dot{n}_{c}}{\delta^{*} F^{*}} & =\int_{0}^{\theta_{g l}} \frac{3 n_{0}-2}{n_{0}-1} \frac{\mathrm{d} \theta}{(\cos \theta)^{n_{0}-1}} \\
& +\int_{\theta_{g l}}^{\frac{\pi}{2}}\left(\frac{3 \cos \theta}{\cos \theta_{g l}}+\frac{1}{n_{0}-1}\right) \frac{\mathrm{d} \theta}{\left(\cos \theta_{g l}\right)^{n_{0}-1}} \\
& -\frac{n_{1}-n_{0}}{\left(n_{1}-1\right)\left(n_{0}-1\right)}\left(\frac{w}{3 \delta^{*}}\right)^{n_{0}-1} \int_{0}^{\frac{\pi}{2}} \mathrm{~d} \theta
\end{aligned}
$$

We note that angles below but close to $\theta_{g l}$ are dominant in the first integral. This justifies our having assumed $\delta_{m}(\theta)<\delta^{*} \quad$ because, from Eq. (8), $\delta_{m} \sim \delta_{g l}=1 / 3 \sqrt{4 / \pi} \sqrt{w h}$, which is typically a few hundred microns; e.g. about $375 \mu \mathrm{m}$ for $w=20 \mathrm{~mm}, h=50 \mu \mathrm{m}$. 


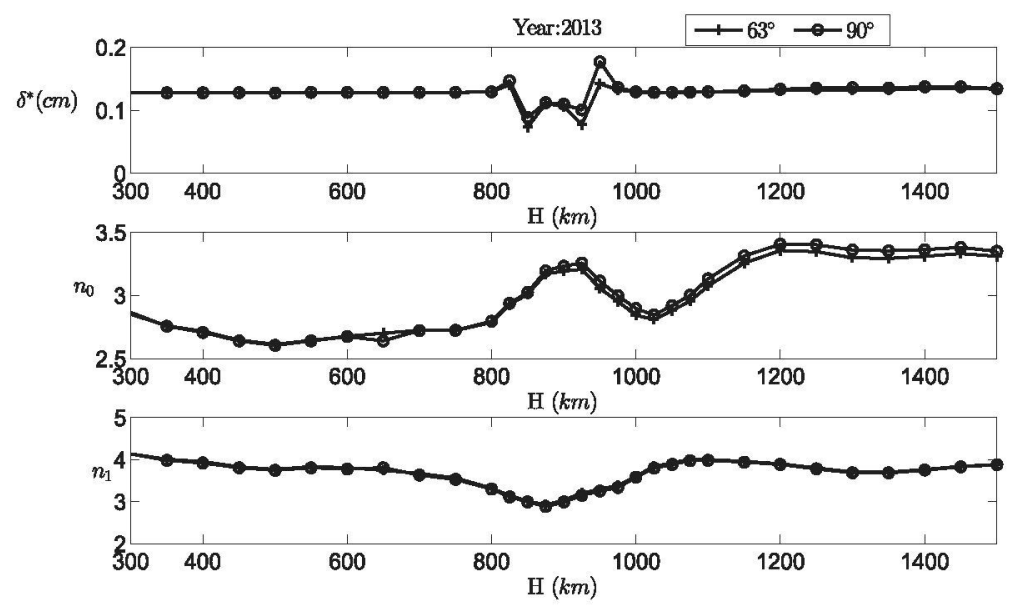

(a) ORDEM2000

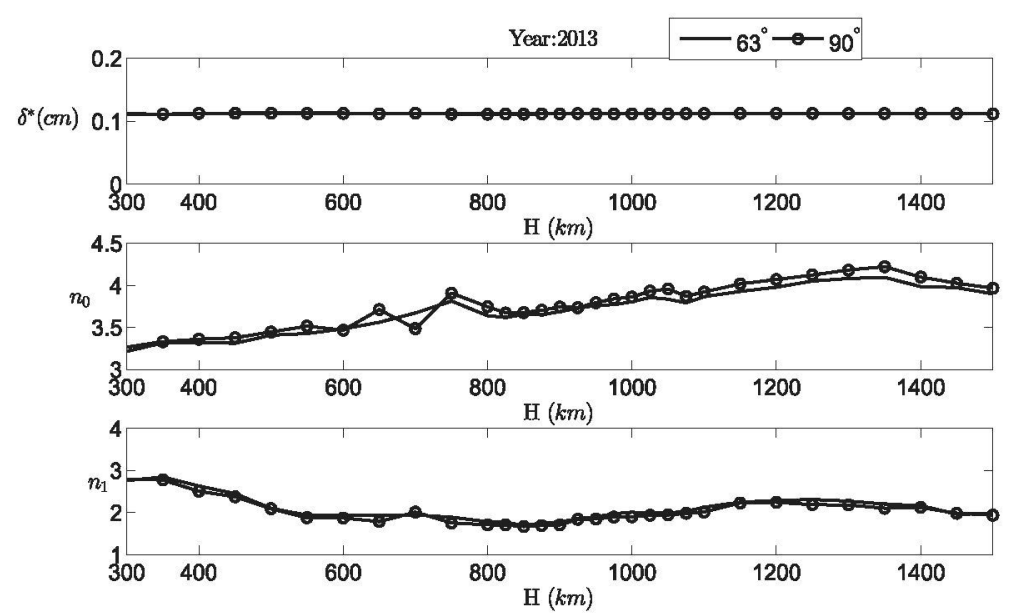

(b) MASTER2009

Fig. 3. Variation of slopes $n_{0}, n_{1}$ and $\delta^{*}$ with altitude, for year 2013

For such particle size, orbital debris flux values are up to one order of magnitude larger than meteoroid flux, except at the few hundred kilometer altitudes (Pardini et al., 2009).

Then, using $\pi / 2-\theta_{g l} \ll 1$ and $n_{0}-1>1$ (as seen in Fig. 3), we define $\cos \theta \equiv v \cos \theta_{g l}$ to find,

$$
\begin{aligned}
\int_{0}^{\theta_{g} l} \frac{\mathrm{d} \theta}{(\cos \theta)^{n_{0}-1}} & \propto \int_{1}^{1 / \cos \theta_{g i}} \frac{\mathrm{d} v}{v^{n_{0}-1} \sqrt{1-\left(\cos \theta_{g l}\right)^{2} v^{2}}} \\
& \simeq \int_{1}^{\infty} \frac{\mathrm{d} v}{v^{n_{0}-1}}=\frac{1}{n_{0}-2}
\end{aligned}
$$

Eq. (14) finally simplifies to,

$$
\dot{n}_{c}=\delta^{*} F^{*}\left[\frac{3 n_{0}+2}{\pi\left(n_{0}-2\right)}\left(\frac{3 \delta^{*}}{w}\right)^{n_{0}-1}\left(\sqrt{\frac{\pi}{4}} \frac{w}{h}\right)^{n_{0}-2}-\frac{n_{1}-n_{0}}{\left(n_{1}-1\right)\left(n_{0}-1\right)}\right]
$$

\section{Discussion of results}

The present model of fatal impact rate, as shown in Eq. (15), exhibits a dependence predominantly on tape dimensions and orbit altitude as $i_{c}(w, h / w, H)$. Eq. (15) will allow simple deorbit calculations for survival. For MASTER2009, given the $n_{0}>n_{1}$ data, the last term in Eq. (15) is negative (Fig. 3 and Fig. 4a), mostly contrary to the case of ORDEM2000. A series of figures have been plotted for fatal impact rate $i_{c}$, calculated from both our simple approximation Eq. (15) full numerical calculations using Eq. (1a) for both ORDEM2000 and MASTER2009, where results obtained using the former shows higher fatal impact rate as its predicted flux $F^{*}$ is about one order of magnitude larger (Fig. 4b), as mentioned earlier. Figs. 5 and 6, for ORDEM2000 and MASTER2009 respectively, show $\dot{n}_{c}$ versus $H$ at two different orbital inclinations $63^{\circ}$ and $90^{\circ}$ for a fixed thickness $h=50 \mu \mathrm{m}$, and four values of width $w$, typically lying within or close to the range of OML validity (Sanmartin and Estes, 1999; Estes and Sanmartin, 2000), for a definite time interval $\Delta t=1$ year (year 


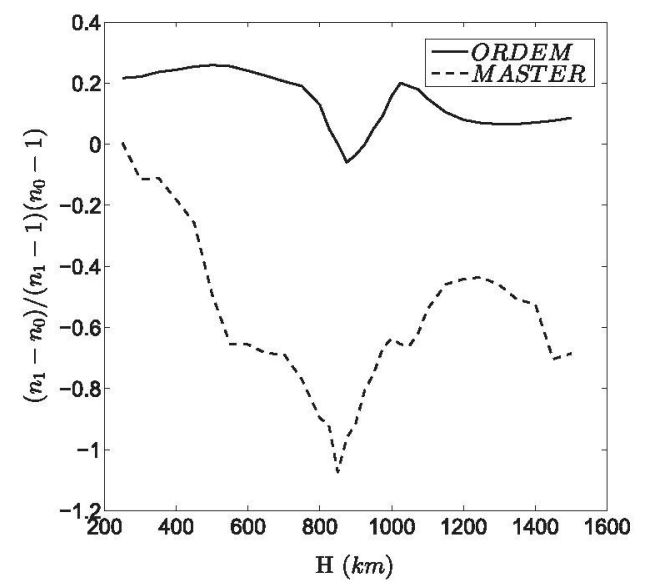

(a) $\left(n_{1}-n_{0}\right) /\left(n_{1}-1\right)\left(n_{0}-1\right)$ vs $H$

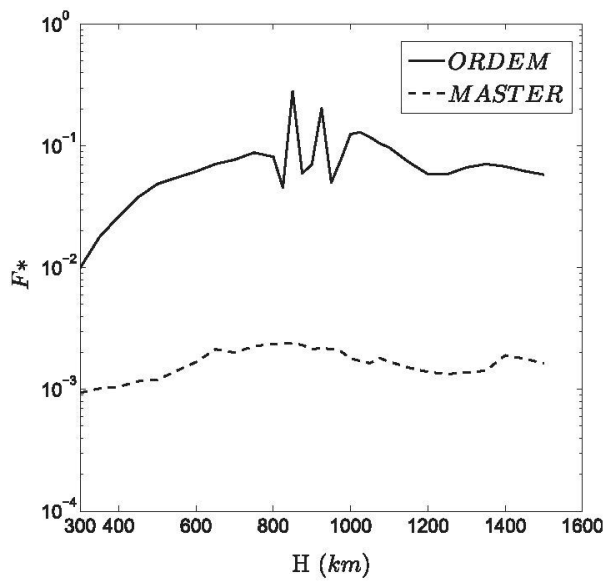

(b) $F^{*}$ vs $H$

Fig. 4. Variation of $\left(n_{1}-n_{0}\right) /\left(n_{1}-1\right)\left(n_{0}-1\right)$ and $F^{*}$ with altitude.
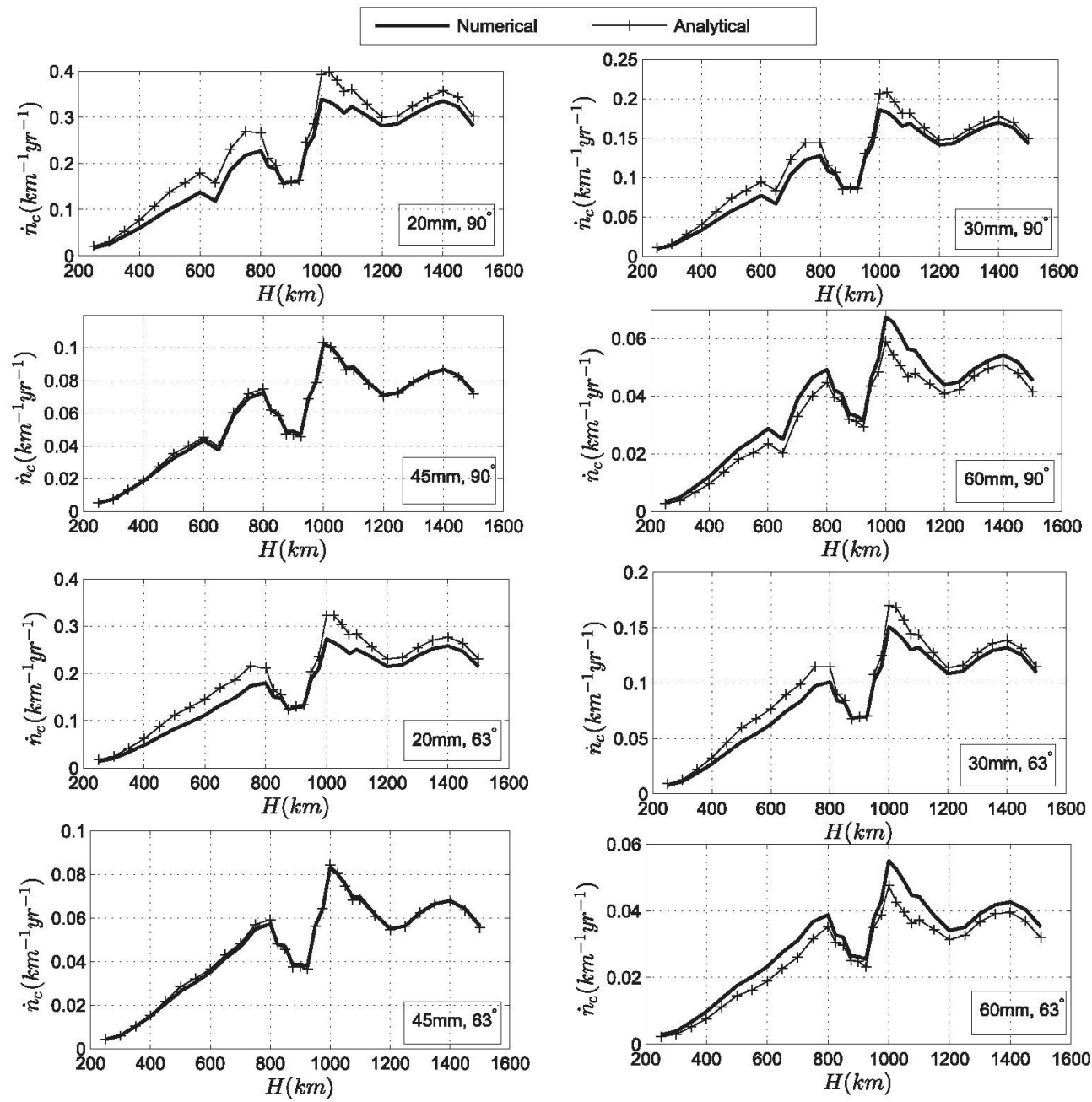

Fig. 5. Analytical and numerical results for fatal impact rate vs. altitude, for different tape width ( $w=20,30,45,60 \mathrm{~mm}$ ), thickness $h=50 \mu \mathrm{m}$ and orbit inclinations $\left(i=90^{\circ}, 63^{\circ}\right)$ for ORDEM2000 data, epoch 2013.

2013). In all four cases the fatal impact rate $\dot{n}_{c}$ decreases as $w$ increases. From Eq. (15) it is also verifiable that $n_{c}$ decreases with the increment of thickness $h$ as well.
The accuracy of the model, when compared with fully numerical computations from the NASA and ESA flux data, is quite reasonable. In the ORDEM2000 case our 


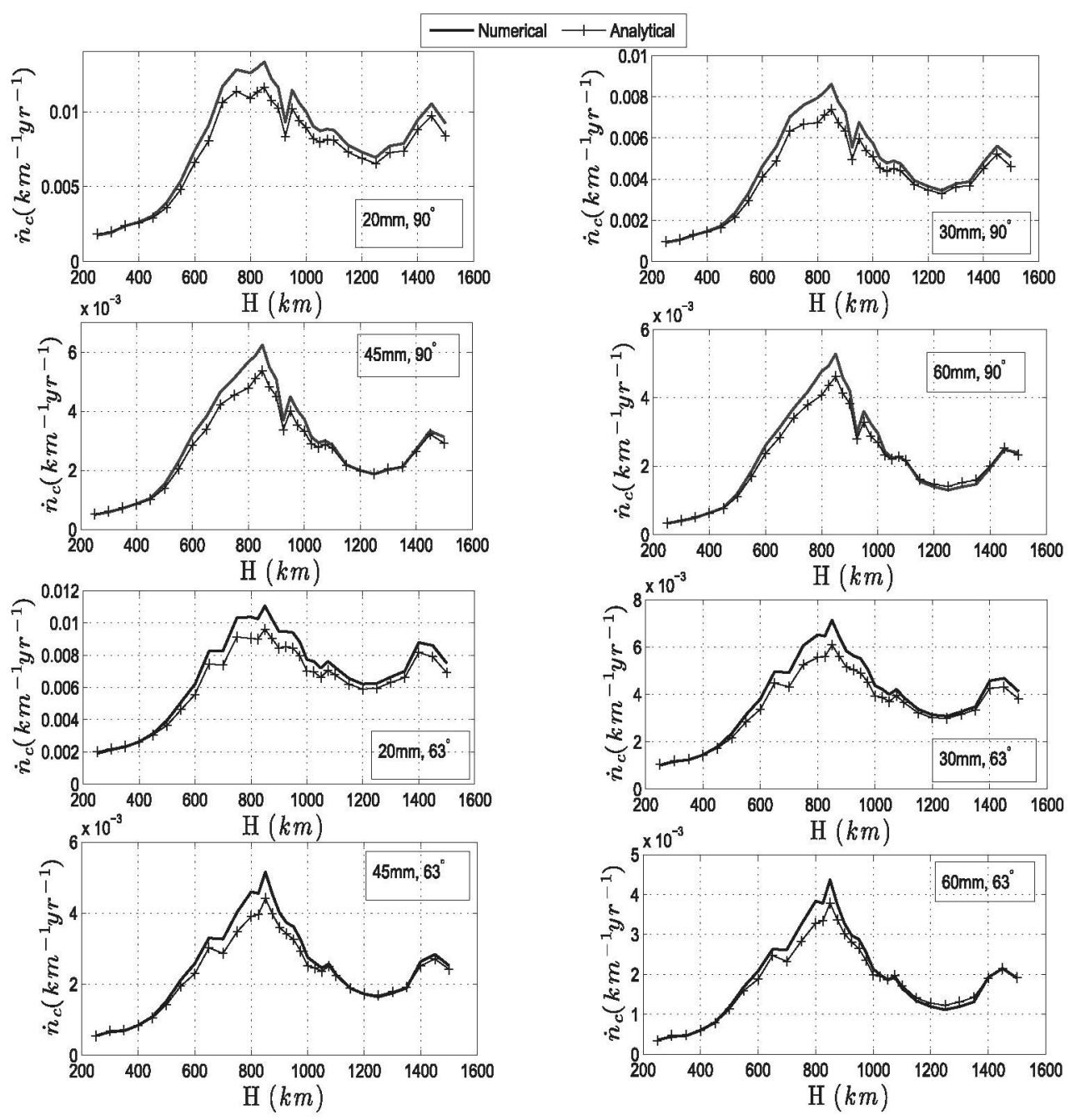

Fig. 6. Analytical and numerical results for fatal impact rate vs altitude, for different tape width $(w=20,30,45,60 \mathrm{~mm})$, thickness $h=50 \mu \mathrm{m}$ and orbit inclinations $\left(i=90^{\circ}, 63^{\circ}\right)$ for MASTER2009 data, epoch 2013.

model overestimates and underestimates results for narrow and wide tapes respectively. Maximum deviation may reach about $12 \%$. In the MASTER2009 case the model underestimates numerical results throughout, at most by $10 \%$. For ORDEM2000 data, since $\delta_{g l} \propto \sqrt{w h}$ approaches $1 \mathrm{~mm}$ and the range $\delta<1 \mathrm{~mm}$ loses relevance, our simple results decrease relative to numerical values being almost coincidental at $w=45 \mathrm{~mm}, h=50 \mu \mathrm{m}$ (Fig. 5). For MASTER2009 data no such dependency is found. Typically, then, our calculations involve debris size not much below $1 \mathrm{~mm}$ to around $1 \mathrm{~cm}$, having flux sensibly larger than micrometeoroid flux except at the lowest altitudes.

\section{Conclusions}

The present work considers debris flux at 32 different LEO altitudes and calculates the fatal impact rate at each altitude for two orbit inclinations. Using NASA and ESA debris flux, our simple analytical formulation allows quite accurately to calculate the tape fatal impact rate per unit time and unit length as a function of tape dimensions $(w, h)$ and orbit parameters (altitude $H$ and inclination $i$ ). Further, this simple model will be useful for preliminary design on tape dimensions ( $w, h$, and $L$ ) depending on desired deorbit mission conditions (e.g. satellite mass, initial altitude). Results are more optimistic for MASTER2009, which is almost 8 to 35 times lower than ORDEM2000, varying on altitude.

\section{Acknowledgments}

This work was supported by a Universidad Politecnica de Madrid Research Grant (RR01/2009) and by the European Commission FP7 Space Project 262972.

\section{References}

Ahedo, E., Sanmartin, J.R., 2002. Analysis of bare-tether systems for deorbiting LEO satellites. J. Spacecraft Rockets 39 (2), 198-205.

Estes, R.D., Sanmartin, J.R., 2000. Cylindrical Langmuir probes beyond the orbital-motion-limited regime. Phys. Plasmas 7, 4320. http:// dx.doi.org/10.1063/1.1288400.

Flegel, S., Gelhaus, J., Wiedemann, C., Vrsmann, P., Oswald, M., Stabroth, S., Klinkrad, H., Krag, H., 2009. The MASTER-2009 space 
debris environment model. In: Proc. 5th European Conference on Space Debris (ESA SP-672, July 2009), Darmstadt, Germany, 30 March-2 April, 2009.

Forward, R.L., Hoyt, R.P., Uphoff, C., 1998. The terminator tether: a near-term commercial application of the NASA/MSFC ProSEDS experiment. In: Proceedings of the Tether Technology Interchanging Meeting, NASA CP-1998-206900, Marshall Space Flight Center, Huntsville, Alabama, USA, pp. 109.

Francesconi, A., Giacomuzzo, C., Kibe, S., Nagao, Y., Higashide, M., 2012. Effects of high-speed impacts on CFRP plates for space applications. Adv. Space Res. 50 (5), 539-548. http://dx.doi.org/ 10.1016/j.asr.2012.05.012

Gilchrist, B., Lim, B., Meckol, N., Masse, R., Williams, S., 2002. The use of electrodynamic tethers for orbit maintenance and deorbit of large spacecraft: a trade study of the NASA GLAST mission. In: Proc., 38th AIAA/ASME/SAE/ASEE Joint Propulsion Conference Meeting \& Exhibit, Paper AIAA 2002-4044, Indianapolis, IN, 7-10 July 2002

Hastings, D., Garrett, H., 1996. Spacecraft Environment Interactions. Cambridge University Press, pp. 251-252.

Hayashida, K.B., Robinson, J.H., 1992. Single Wall Penetration Equations, National Aeronautics and Space Administration, George C. Marshall Space Flight Center, AL, 1992.

Kessler, D.J., 2000. Presentation at NASA Marshall Space Flight Center, Huntsville, AL, 2000.

Kessler, D.J., Cour-Palais, B.G., 1978. Collision frequency of artificial satellites: the creation of a debris belt. J. Geophys. Res. : Space Phys. 83 (A6), 2637-2646

Khan, Shaker, B., Sanmartin, J.R., 2013. Survival probability of round and tape tethers against debris impact. J. Spacecraft Rockets 50 (3), 603-608. http://dx.doi.org/10.2514/1.A32383.

Liou, J.C., Matney, M.J., Anz-Meador, P.D., Kessler, D., Jansen, M., Theall, J.R., 2002. "The New NASA Orbital Debris Engineering Model ORDEM2000. NASA STI/Recon Technical Report N 2, 51086.

Nishimine, A., 2002. Debris collision analysis for tethers. In: 23rd International Symposium on Space Technology and Science, Paper ISTS 2002-s-29, Matsue, Japan, May 23-June 2, 2002, pp. 2524-2529.
Pardini, C., Hanada, T., Krisko, P.H., Anselmo, L., Hirayama, H., 2007. Are de-orbiting missions possible using electro-dynamic tethers? Task review from the space debris prospective. Acta Astronaut. 60 (10-11), 916-929.

Pardini, C., Hanada, T., Krisko, P.H., 2009. Benefits and risk of using electro-dynamic tethers to deorbit spacecraft. Acta Astronaut. 64 (5), 571-588.

Pearson, J., Carroll, J., Levin, E., Oldson, J., Hausgen, P., 2003. "Overview of the electrodynamic delivery express (EDDE)". In: Proc: 39th AIAA/ASME/SAE/ASEE Joint Propulsion Conference Meeting \& Exhibit, Paper AIAA 2003-4790, Huntsville, AL, 20-23 July 2003.

Ryan, S., Christiansen, E.L., 2010. Micrometeoroid and Orbital Debris (MMOD) Shield Ballistic Limit Analysis Program, NASA/TM-2009214789, National Aeronautics and Space Administration Johnson Space Center Houston, TX 77058, February 2010.

Sanmartin, J.R., Estes, R.D., 1999. The orbital-motion-limited regime of cylindrical Langmuir probes. Phys. Plasmas 6, 395. http://dx.doi.org/ $10.1063 / 1.873293$.

Sanmartin, J.R., Ahedo, E., Martinez-Sanchez, M., 1992. An anodeless tether generator. Physics of Charged Bodies in Space Plasma. Societa Italiana de Fisica, Bologna, Italy, pp. 241-248.

Sanmartin, J.R., Martinez-Sanchez, M., Ahedo, E., 1993. Bare wire anodes for electrodynamic tethers. J. Propul. Power 9 (3), 353-360.

Simon, C.G., Hunter, J.L., Griffis, D.P., Misra, V., Ricks, D.A., Wortman, J.J., Brownlee, D.E., 1993. Elemental analyses of hypervelocity microparticle impact sites on interplanetary dust experiment sensor surfaces. In: LDEF: 69 Months in Space. Part 4: Second PostRetrieval Symposium, vol. 1, April 1993, pp. 677-692.

Van der Heide, E.J., Kruijff, M., 2001. Tethers and debris mitigation. Acta Astronaut. 48 (5), 503-516.

Vannaroni, G., Dobrowolny, M., De Venuto, F., 1999. Deorbiting with electrodynamic tethers: comparison between different tether configurations. Space Debris 1 (3), 159-172. 